\title{
Mass transfer of anti-cancer drug delivery to brain tumours by a multiple emulsion-based implant
}

\author{
Ewa Dluska ${ }^{1}$, Agnieszka Markowska-Radomska ${ }^{2}$, Agata Metera $^{2}$, Leszek Rudniak ${ }^{2}$, and \\ Konrad Kosicki ${ }^{3}$ \\ ${ }^{1}$ Politechnika Warszawska \\ ${ }^{2}$ Warsaw University of Technology \\ ${ }^{3}$ University of Warsaw
}

June 26, 2021

\begin{abstract}
The advanced use of a pH-responsive biomaterial-based injectable liquid implant for effective chemotherapeutic delivery in glioblastoma multiforme brain (GBM) tumour treatment is presented. As an implant, we proposed a water-in-oil-in-water multiple emulsion with encapsulated doxorubicin. The effectiveness of the proposed therapy was evaluated by comparing the cancer cell viability achieved in classical therapy (chemotherapeutic solution). The experimental study included doxorubicin release rates and consumption for two emulsions differing in drop sizes and structures in the presence of GBM-cells (LN229, U87 MG), and a cell viability. The results showed that the multiple emulsion implant was significantly more effective than classical therapy when considering the reduction in cancer cell viability: $85 \%$ for the emulsion-implant, and only $43 \%$ for the classical therapy. A diffusion-reaction model was adapted to predict doxorubicin release kinetics and elimination by glioblastoma cells. CFD simulations confirmed that the drug release kinetics depends on multiple emulsion structures and drop sizes.
\end{abstract}

Mass transfer of anti-cancer drug delivery to brain tumours by a multiple emulsion-based implant

Ewa Dluska ${ }^{1}$

Agnieszka Markowska-Radomska ${ }^{1}$

Agata Metera ${ }^{1}$

Leszek Rudniak ${ }^{1}$

Konrad Kosicki ${ }^{2}$

${ }^{1}$ Faculty of Chemical and Process Engineering, Warsaw University of Technology, Warynskiego 1, 00645 Warsaw, Poland, ewa.dluska@pw.edu.pl, agnieszka.markowska@pw.edu.pl, agata.metera@pw.edu.pl, leszek.rudniak@pw.edu.pl

${ }^{2}$ Faculty of Biology, Institute of Genetics \& Biotechnology, University of Warsaw, Miecznikowa 1, 02-096 Warsaw, Poland, konrado@biol.uw.edu.pl

\section{Corresponding author:}

Ewa Dluska

ewa.dluska@pw.edu.pl

Faculty of Chemical and Process Engineering, 
Warsaw University of Technology,

Warynskiego 1, 00-645 Warsaw, Poland.

Tel: +48222346296

Fax: +48228251440

\section{Abstract}

The advanced use of a pH-responsive biomaterial-based injectable liquid implant for effective chemotherapeutic delivery in glioblastoma multiforme brain (GBM) tumour treatment is presented. As an implant, we proposed a water-in-oil-in-water multiple emulsion with encapsulated doxorubicin. The effectiveness of the proposed therapy was evaluated by comparing the cancer cell viability achieved in classical therapy (chemotherapeutic solution). The experimental study included doxorubicin release rates and consumption for two emulsions differing in drop sizes and structures in the presence of GBM-cells (LN229, U87 MG), and a cell viability. The results showed that the multiple emulsion implant was significantly more effective than classical therapy when considering the reduction in cancer cell viability: $85 \%$ for the emulsion-implant, and only $43 \%$ for the classical therapy. A diffusion-reaction model was adapted to predict doxorubicin release kinetics and elimination by glioblastoma cells. CFD simulations confirmed that the drug release kinetics depends on multiple emulsion structures and drop sizes.

\section{INTRODUCTION}

Drug delivery in the treatment of the central nervous system diseases - CNS (brain tumours, trauma, infections, neurodegenerative problems, amongst others) requires passing through, or bypassing, the blood-brain barrier (BBB). The methods of drug administration to the CNS can be divided into three main groups: invasive techniques, non-invasive techniques and alternative routes. ${ }^{1,2,3}$ The non-invasive techniques explore approaches in which pharmaceuticals are re-engineered to cross the BBB via: (i) chemical methods (lipophilic analogues, prodrugs, enzymatic reactions or chemical bonding of drug molecules with transport facilitated molecules), or (ii) biological methods (drug attachment to proteins specific for receptors responsible for transport across the BBB, transport vectors or barrier-crossing peptides). In addition, nanoparticles, dendrimers, liposomes, micelles, micro/nanoemulsions, including targeted drug delivery systems, and stimuli-responsive functional biomaterials in the drug delivery area, are used to cross the BBB..$^{4,5,6}$ The invasive techniques include: (i) local surgical treatment combined with adjuvant therapy (intracerebral polymer implants or microchips, intraventricular/intrathecal or interstitial drug delivery, biological tissue delivery) or (ii) controlled BBB damage with drug delivery (e.g. convection-enhanced drug delivery, osmotic or ultrasound disruption of the BBB). Surgical treatment in combination with radio and chemotherapy, in the case of brain tumours, plays a fundamental role in neurooncology. If possible, tumours should be removed completely. In most cases, only part of the tumour is surgically removed, and the remainder is irradiated or subjected to chemotherapy for destruction. The alternative methods bypass the cardiovascular system and include transnasal administration of drugs or iontophoretic delivery. Modern medicine responds to the needs of the patient based on various strategies including mathematical modelling for predicting the effect of chemotherapy on cancer cells. The approaches to simulating cancer interaction with therapy can generally range from (i) cancer growth models, then (ii) mass transfer models, which combine drug release, transport, and elimination in tissue, to (iii) cell-based models at the molecular level. The first group includes models of tumour growth/volume change based on the analysis of cell proliferation and cell death, along with the structure of an avascular (solid) tumour (homogeneous or heterogeneous), and a vascular heterogeneous tumour, including tumourinduced angiogenesis. ${ }^{7,8}$ The second group - reaction-diffusion/convection models are related to transport mechanisms for delivering drugs to the tumour and drug elimination in tissue by chemical reaction. These models provide macroscopic descriptions of the system based on space- and time-dependent variables such as drug concentration distribution, pressure in the tumour environment, and drug release rates. ${ }^{9,10,11}$ The third group represents discrete cell-based models describing the dynamics of cancer cells via individual cell behaviours within the tumour tissue based on the transformation and transport of substances to the cell at the molecular level. Models that treat cells individually can combine descriptions at the subcellular and 
cellular levels, with a macroscopic description of the tumour environment. This extended approach via, e.g. the lattice-based method or cellular automata, creates new hybrid computational models for simulating the cancer intercellular adhesion and invasion process. ${ }^{12,13,14,15,16}$ In the present study, a diffusion-reaction model was adapted to predict the accurate chemotherapeutic concentration after its in vitro release from a liquid implant in the presence of glioblastoma multiforme cells. Glioblastoma multiforme (GBM) is a primary malignant tumour of the central nervous system with one of the worst prognoses. Despite huge progress in the field of oncology, the median survival rate for patients after diagnosis is less than 2 years. ${ }^{2}$ As the liquid implant, we proposed multiple emulsions $\mathrm{W} / \mathrm{O} / \mathrm{W}$ (water-in-oil-in-water) type with encapsulated doxorubicin (chemotherapeutics) in the internal droplets, Fig. 1. Multiple emulsions are complex dispersed systems with a hierarchical structure of droplets of the first liquid dispersed in larger drops of a second immiscible liquid, which is a dispersion medium for smaller droplets. The larger drops are then dispersed either in the continuous phase of the first liquid (double emulsion) or in other still larger drops of the first or other immiscible liquid and so on (a multiple emulsion). A multiple emulsion can be then a double, triple, quadruple, quintuple, or even more structured system. These dispersed systems offer a wide range of possible applications in separation processes and environmental protection (alternative fuel), pharmaceuticals, and medicine, especially for the encapsulation and controlled release of active ingredients (drugs, living cells, antigen delivery, cosmetics, food). ${ }^{17,18,19,20,21,22,23,24,25}$ Controlling by multiple emulsions is achieved through the size and physicochemical parameters of drops forming liquid-permeable membranes separating the internal droplets from the continuous external phase. The proposed method involves inserting the liquid implant intraoperatively into the cavity after surgical resection of the tumour, Fig. 1. This method is a bypassing of the BBB and belongs to the group of adjuvant treatments. Administering the drug in the form of multiple emulsions is intended to support the treatment, i.e. the cytotoxic effect on the tumour cells remaining after the surgery to prevent the recurrence of the tumour. The chemotherapeutic agent is released from the liquid implant into the GBM environment in a manner controlled by the $\mathrm{pH}$ of the tumour environment and also the drop size, structure, or physicochemical parameters of the emulsion (viscosity, density). The external phase of the emulsion contains a biopolymer (sodium carboxymethylcellulose) which, depending on the $\mathrm{pH}$, changes the conformation of the chains and the viscosity, thus affecting the release rates. Such properties of the polymer are exploited in this concept for controlled release under the acidic tumour microenvironment. The use of the implant in a liquid form also reduces the risk of mechanical damage to healthy tissue during the intraoperative insertion in comparison to a solid implant. The paper aims to find optimal brain cancer treatment based on locally controlled chemotherapeutic release from an emulsion-based implant, including numerical simulations using a diffusion-reaction model for drug transport and consumption. To evaluate this model, the paper includes a comparison of these simulations with experimental data on anti-cancer drug release and consumption for two emulsions differing in drop sizes and structures. Also, cancer cell viability was investigated in comparison with classical chemotherapy involving cells treated with a drug in a solution.

\section{MATERIALS AND METHODS}

2.1 Preparation and characterization of multiple emulsions with anti-cancer drug (drop size, viscosity)

Multiple/double emulsions W1/O/W2 with doxorubicin hydrochloride - DOX (anti-cancer drug) were prepared in a Couette-Taylor flow (CTF) apparatus where liquid phases were intensively mixed due to rotational and axial flows. The emulsions preparation conditions are shown in Table 1. The detailed procedure for the preparation of the emulsion can be found in the previous authors' works ${ }^{26,27,28}$. In short, the internal water phase (W1) with DOX and soybean oil as an organic membrane phase (O) were introduced in the inlet cross-section of the CTF apparatus and intensively mixed to create simple emulsions $\mathrm{W} 1 / \mathrm{O}$. Then, after introducing the water phase (W2) to the simple emulsions in the middle section, double emulsions $\mathrm{W} 1 / \mathrm{O} / \mathrm{W} 2$ were formed. The CTF apparatus provides high mass transfer parameters and uniform shear flow, contributing to the high encapsulation efficiency and formation of stable emulsions. ${ }^{28,29,30}$ The structures of the obtained emulsions were analyzed using an Olympus BX60 optical microscope, with Olympus SC50 digital camera (Olympus, Japan), and image analysis software, Image Pro Plus 4.5 (Media Cybernetics, USA). For each of the double emulsions, at least 800 drops of the membrane phase and 1000 drops of the internal phase were measured. Then drop sizes distributions were determined and the average drop sizes: 
the Sauter mean diameter of the internal $\left(\mathrm{d}_{32}\right)$ and membrane phase $\left(\mathrm{D}_{32}\right)$ drops. Also, the volume fractions of the internal phase drops in the membrane phase drops were determined (packing volume fraction). The fluorescence spectrofluorometer FLUOstar Optima (BMG LABTECH, Germany) was used to measure the concentration of the chemotherapeutic agent in the emulsion continuous phase to determine the encapsulation efficiency of the DOX in the internal phase droplets. The emulsions rheological tests were performed with a RheolabQC rotational rheometer (the measuring system of concentric cylinder geometry - gap size: $1.64 \mathrm{~mm}$, length $60 \mathrm{~mm}$, cone angel: $120^{\circ}$, ratio of radii: 1.08 , range of shear rate: $1-1500 \mathrm{~s}^{-1}, 37 \mathrm{degC}$, Anton Paar, Austria). Two stable soybean oil-based emulsions, differing in the internal structure of the drops, with pH-responsive biopolymer (sodium carboxymethylcellulose - CMC-Na) in the external phase were selected for further experiments (Table 1). The emulsions were characterized by a high encapsulation efficiency of (DOX) (>95\%), calculated based on the difference in DOX concentration introduced to the CTF apparatus and in the external phase of created multiple emulsions according to the procedure ${ }^{26}$. All compounds used to prepare emulsions were supplied by Sigma Aldrich. Detailed data on the composition and preparation conditions of the emulsions can be found in Table 1.

\subsection{Glioblastoma model cell-lines culturing}

The in vitro studies on cell viability and release of the anti-cancer/cytostatic agent from multiple emulsions were conducted for selected tumour cell lines of glioblastoma multiforme: U87 MG, LN229. The cell lines were procured from the Institute of Biochemistry and Biophysics PAS (Poland). The cells were cultured on $10 \mathrm{~cm}$ dishes (BD-Falcon, USA) to 80-90\% confluence in DMEM medium with high glucose and L-glutamine (HyClone, Poland), with 10\% fetal bovine serum (FBS; Gibco, Poland) and 1\% penicillin/streptomycin antibiotics (Life Technologies, Poland) in an incubator (37degC; $5 \% \mathrm{CO}_{2}$ ). The cells were passaged 24 hours prior to release and cytotoxicity experiments (cells were first rinsed with phosphate-buffered saline-PBS buffer (Lab Empire, Poland), then trypsinized by $0.25 \%$ trypsin and $0.1 \%$ EDTA (HyClone, Poland).

\subsection{Release of anti-cancer drug from multiple emulsions}

The in vitro release experiments of the anti-cancer/cytostatic drug (DOX) from multiple emulsions were carried out in the systems with and without glioblastoma cells (U87 MG, LN229) to determine the drug consumption by cells. Standard 12-well plates were used, 4000 cells/wells were seeded $24 \mathrm{~h}$ before the release experiment and placed in the incubator (temperature: $37 \mathrm{degC}, 5 \% \mathrm{CO}_{2}$, release volume: $1 \mathrm{~cm}^{3}$ ). The release process was analyzed for a specific concentration of DOX encapsulated in the emulsion $(0.1 \mu \mathrm{M}, 0.2 \mu \mathrm{M})$. The target concentration of DOX was obtained by diluting the emulsions with PBS buffer of $\mathrm{pH}=7.4$ (1:100, 1:50 - volume of emulsion to PBS buffer). At certain time intervals during the $24 \mathrm{~h}$ experiment with cells and $96 \mathrm{~h}$ without cells, the entire volume of the diluted emulsion was taken from each well $\left(1 \mathrm{~cm}^{3}\right)$ and filtered through a hydrophilic syringe filter (nylon filter membrane, $0.2 \mu \mathrm{m}$ ). The DOX concentration in the samples was determined using a fluorescence spectrofluorometer - FLUOstar Optima (BMG LABTECH, Germany), measured at a wavelength of Ex $488 \mathrm{~nm} / \mathrm{Em} 593 \mathrm{~nm}$ (excitation/emission). Prior to the release studies, the effect of PBS buffer at $\mathrm{pH} 7.4$ on cell viability was monitored. It was assumed that the relative viability of cells was not lower than $80 \%$. The measurement of cell viability was performed in the presence of cells in PBS buffer for $3 \mathrm{~h}, 6 \mathrm{~h}, 9 \mathrm{~h}, 24 \mathrm{~h}$. The measurements were carried out $24 \mathrm{~h}$ after re-positioning the cells in the full culture medium, according to the procedure described in the cytotoxicity studies. These results determined the limitation of the experiment time, when the cells were surely in good condition, to the maximum time of 7h. Measurements of concentration after 24 hours in a system with cells were excluded due to cell viability

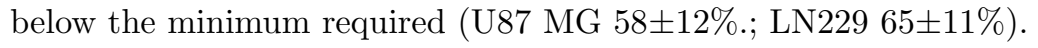

\subsection{Elimination rate constant of anti-cancer drug in the presence of cancer cells}

The doxorubicin (DOX) elimination/consumption rate constants by GBM cells (U87 MG, LN229) were determined based on the DOX depletion in the system. The mass fraction of DOX absorbed by cells was calculated as the difference between the concentration of DOX released from the multiple emulsion in a cellfree environment and the presence of cancer cells. The mass fraction of the drug available was determined by subtracting from the value 1 (the mass fraction corresponding to the total drug availability at time $t=0$ ) the 
mass fraction of DOX absorbed (consumed) by cells for a given time. The obtained values were presented as a function: $\ln (1$-mass fraction of consumed DOX) vs time with a linear approximation following the kinetics of the first-order reaction. The drug elimination rate constant was then found as a slope of this function. The drug consumption rate constants and the linear function fit coefficients were determined for each glioblastoma cell line and for the tested DOX concentrations in emulsions DOX-E1 and DOX-E2, which are summarised in Table 2 .

\subsection{Cytotoxicity study of multiple emulsions with DOX}

In vitro cytotoxicity tests were performed using the REDOX test based on Alamar Blue reagent (Invitrogen, USA) according to the manufacturers' instructions. Cells (U87 MG, LN229) were seeded on standard 96-well plates with 400 cells/wells to maintain a proportional number of cells in relation to vessel geometry for release and cytotoxicity studies. After 24 hours from seeding, cells were exposed to a potentially cytotoxic agent (DOX). Cytotoxic effects were determined for the DOX concentrations in the emulsions at $0.1 \mu \mathrm{M}$ and $0.2 \mu \mathrm{M}$. These dosages of DOX were obtained by the dilution of emulsion in a culture medium with the proportions 1:100 and 1:50, respectively. In cytotoxicity studies, the emulsion was diluted with a full culture medium, with a composition identical to that used for the cell culture. In earlier studies, the effect of changes in DOX concentration on U87MG cell viability was checked ${ }^{26}$. The cell viability was determined for cells treated with emulsion, without DOX (E1 and E2 -negative control), emulsion with encapsulated DOX (DOX-E1 and DOX-E2), and DOX solution in full culture medium. The measurements were carried out for the three different times of cancer cell contact with a potentially cytotoxic agent: $24 \mathrm{~h}, 48 \mathrm{~h}, 72 \mathrm{~h}$. After the established contact time, the culture medium was exchanged for a fresh culture medium with Alamar Blue reagent $(1: 10 \mathrm{v} / \mathrm{v})$. After $24 \mathrm{~h}$ and $168 \mathrm{~h}$ the RFUs (relative fluorescence units) were measured at a wavelength of Ex $560 \mathrm{~nm} / \mathrm{Em} 590 \mathrm{~nm}$ (excitation/emission) using a multimode detector (DTX880, Beckman Coulter, Canada). The relative cell viability was calculated as a ratio to that of the untreated control cells. All obtained values are the result of three independent experiments. Each of the parameters was determined in triplicate.

\subsection{Emulsion viscosity measurements}

A RheolabQC rotational viscometer with a double gap measuring cylinder (DG42) (Anton Paar, Austria) was used to study the rheological properties of the emulsions DOX-E1 and DOX-E2, and the external phases of both emulsions. To study multiple emulsions as systems responding to changes in a $\mathrm{pH}$ environment, emulsions and their external phases were diluted before measurement with a phosphate buffer (PBS buffer) at $\mathrm{pH} 7.4$ (alkaline) and $\mathrm{pH} 6.3$ (acidic). Samples were diluted in a volume dilution ratio of emulsion or external phase in PBS buffer at 1:10 (volume of tested sample to PBS buffer).

Measurements were performed for 3 independently prepared samples in the shear rate range 50-2500 $\mathrm{s}^{-1}$ during testing. Samples of emulsions for rheological tests were placed in the measuring cylinder immediately after the emulsification process. The tests were performed after establishing the temperature of the sample at $37^{\circ} \mathrm{C}$. During the measurement, the emulsion structures were microscopically observed to ensure the stability of the emulsions.

\subsection{Statistical analysis}

All data in this study were expressed as a mean \pm standard error $( \pm \mathrm{SD})$ of at least three independent experiments. Each of the parameters/values was determined in triplicate.

2.8 Mathematical model development of drug release from the emulsion-based implant and drug elimination by cancer cells

\section{Model concept and assumptions}

The equation of unsteady state diffusion with a chemical reaction was exploited to describe the release process of a chemotherapeutic drug from the drops of an emulsion, its transport by diffusion to glioblastoma 
cells and drug consumption/elimination by cells, Fig 1 . The mechanisms and steps in the emulsion system and cancer cells environment:

(i) diffusional transport of a drug (doxorubicin-DOX) from internal droplets of W1/O/W2 emulsions to the membrane phase drops through the interface of $\mathrm{W} 1 / \mathrm{O}$;

(ii) diffusional transport of a drug from the membrane phase drops to the external continuous phase through the interface of $\mathrm{O} / \mathrm{W} 2$;

(iii) diffusional transport of a drug in an external environment representing the interstitial fluid of the tissue in which the glioblastoma cells are located;

(iv) elimination/consumption of a drug by the glioblastoma cells (the biological system) via a first-order chemical reaction.

Model assumptions:

- the release of the drug occurs from a population of drops represented by the mean (Sauter) diameter of the drops of the internal $\left(\mathrm{d}_{32}\right)$ and membrane $\left(\mathrm{D}_{32}\right)$ phases of a multiple emulsion;

- neglected mass transfer resistance of the drug in the internal droplets, resulted from a much higher diffusion coefficient in the water phase (internal phase) compared to the organic phase (membrane phase) and the difference in drop sizes $\mathrm{r}_{\mathrm{w}}[?] \mathrm{R}$;

- the mass transfer resistance of a drug in the membrane phase is described by the volumetric mass transfer coefficient-model parameter $\mathrm{k}_{\mathrm{L}} \mathrm{a}$ (equation 2);

- the release of a drug to the external phase takes place after the equilibrium is attained between the drug concentrations in the internal (water) and membrane (organic) phases of multiple emulsion;

- the elimination rate constant of the drug is calculated according to the kinetics of an irreversible first-order reaction ${ }^{9,10}$

- no coalescence of drops, no breakdown, no internal circulation, stable structure of the emulsion $\left(\mathrm{r}_{\mathrm{w}}, \mathrm{R}\right.$ = const.), constant transport, and physicochemical parameters of emulsions.

The model consists of partial differential equations describing drug release and transport coupled with a drug elimination by cancer cells and initial and boundary conditions. Governing equations for a drug mass balance in the emulsion phases and the tumour environment for the spherical coordinate system:

1. Changes in the concentration of a drug (chemotherapeutic agent - doxorubicin (DOX)) in the membrane phase drops of emulsion for 0 [?] $\mathrm{r}$ [?] $\mathrm{R}$

$\frac{\partial C(r, t)}{\partial t}=D_{e} \bullet\left(\frac{1}{r^{2}} \frac{\partial}{\partial r}\left(r^{2} \frac{\partial C(r, t)}{\partial r}\right)\right)+\kappa \bullet\left(\left(\frac{\varphi}{1-\varphi}\right) \bullet\left(m \bullet C_{S}(r, t)-C(r, t)\right)\right)(1)$

$\kappa=k_{L} a=\frac{3 \bullet D_{e}}{r_{w}^{2}} \bullet \frac{\phi^{1 / 3}}{\left(1-\phi^{1 / 3}\right)}(2)$

2. Changes in the concentration of the drug (DOX) in the internal phase droplets for 0 [?] $\mathrm{r}[?] \mathrm{r}_{\mathrm{w}}$

$\frac{\partial C_{S}(r, t)}{\partial t}=\kappa \bullet\left(C(r, t)-m \bullet C_{S}(r, t)\right)(3)$

$m=\frac{C^{*}}{C_{s}^{*}}(4)$

3. Changes in the concentration of the drug (DOX) in the continuous phase, outside the large drops, i.e. in the environment of cancer cells, for $\mathrm{r}>\mathrm{R}$

$\frac{\partial C_{z}(r, t)}{\partial t}=D_{e, z} \bullet\left(\frac{1}{r^{2}} \frac{\partial}{\partial r}\left(r^{2} \frac{\partial C_{z}(r, t)}{\partial r}\right)\right)-k \bullet C_{z}(r, t)(5)$

where $k \bullet C_{z}(r, t)$ represents the rate of drug elimination by cancer cells

Solution of the coupled diffusion/elimination equations requires an initial and several boundary conditions for a spherical coordinate system. 
The initial conditions:

$C(r, t)=C(r, 0)=C_{S}(r, 0) \bullet m=C_{S, 0} \bullet \phi \bullet m$ for $0 \leq r \leq R(6)$

$C_{z}(r, 0)=0$ for $\mathrm{r}>\mathrm{R}(7)$

$C_{S}(r, 0)=0$ for $\mathrm{r}>\mathrm{R}(8)$

The boundary conditions at the interface:

for $\mathrm{r}=\mathrm{R}$

$\left.\left.D_{e} \frac{\partial C(r, t)}{\partial r}\right\rceil_{r=R}=D_{e, z} \frac{\partial C_{z}(r, t)}{\partial r}\right\rceil_{r=R}(9)$

$C(R, t)=C_{z}(R, t) \bullet n(10)$

$n=\frac{C^{*}}{C_{z}^{*}}(11)$

for $\left.\mathrm{r}=0 D_{e} \frac{\partial C(r, t)}{\partial r}\right\rceil_{r=0}=0(12)$

for r-[?] $C_{z}=0$ (13)

C (r, t) - spatiotemporal drug (DOX) concentration function of change in the membrane phase drops of multiple emulsion, $\mathrm{r}$ - radius, $\mathrm{t}$ - release time, $\mathrm{C}_{\mathrm{z}}(\mathrm{r}, \mathrm{t})$ - spatiotemporal drug concentration function in the external phase of multiple emulsion, $\mathrm{C}_{\mathrm{s}}(\mathrm{r}, \mathrm{t})$ - spatiotemporal drug concentration function equivalent to the concentration in the internal droplets of multiple emulsion, $\mathrm{C}_{\mathrm{S}, 0}$ - the initial concentration of drug in the internal droplets, $\mathrm{D}_{\mathrm{e}}$ - effective diffusion coefficient of drug in the membrane phase drops, $\mathrm{D}_{\mathrm{e}, \mathrm{z}^{-}}$effective diffusion coefficient of drug in the external phase, $\mathrm{k}$ - the first order elimination rate constant of the drug by GBM cells, $\mathrm{k}_{\mathrm{L}} \mathrm{a}$ - volumetric mass transfer coefficient of drug in the membrane phase drops $\left(\mathrm{k}_{\mathrm{L}} \mathrm{a}=x\right)$, $\mathrm{m}$ - equilibrium drug partition coefficient between the membrane phase and the internal phase of emulsion, $\mathrm{n}$ - equilibrium drug partition coefficient between the membrane phase and the external phase of emulsion, $\mathrm{C}^{*}$ - equilibrium concentration, $\mathrm{r}_{\mathrm{w}}$ - radius of the internal emulsion droplets, $\mathrm{R}$ - radius of the membrane phase drop of emulsion, $\varphi$ - volume fraction of the internal droplets in the membrane phase drops of multiple emulsion (packing volume fraction).

\section{Model solution}

The model equations were solved numerically using ANSYS Fluent v. 2020R2 software and the finite volume method (FVM). A computational domain was defined, considering the drops and the external fluid in which the glioblastoma cells absorb (consume) the drug (DOX) released from drops of emulsions DOX-E1 and DOX-E2. Based on the analysis of the number of drops per unit volume (drops + the external surrounding fluid), the simulation domain was determined as the relationship between the radius of the drops and the radius of the external fluid into which the drug was released. Due to the spherical geometry and axial symmetry, the computational domain was defined and discretised in cylindrical coordinates.

\section{RESULTS AND DISCUSSION}

3.1 The controlled anti-cancer drug release from the pH-responsive multiple emulsion and elimination by GBM cells: experiments and simulations

The experimental data and simulations of the release rates of DOX from multiple emulsions DOX-E1 and DOX-E2 in the cancer cell environment (LN229 and U87 MG) are presented in Fig. 2 (c, d, f, g) as a cumulative mass of DOX released vs time for two DOX concentrations in emulsions.

The experimental results showed that the release rates of DOX are influenced by the drop sizes and emulsion structures in the presence of the tumour environment characterised by acidic $\mathrm{pH}$. The diffusional release of DOX from the emulsion with smaller drops (DOX-E2) was faster than those from the emulsion with larger drops (DOX-E1). Faster release from DOX-E2 resulted from a larger interfacial related to the smaller drop sizes of these emulsions. In addition, the internal structure of emulsion DOX-E2 (single droplet in a 
drop-Fig. 2b) shortened the DOX diffusion path to the drops' interface and outside, and so thus led to a faster release compared to the emulsion DOX-E1, structured as many internal droplets in a drop (Fig. 2a). Our previous results related to the release rates of $\mathrm{DOX}$ from multiple emulsions in $\mathrm{pH}=6.3$ simulating the biological system of cancer cells confirmed a faster release of DOX in comparison with $\mathrm{pH}=7.4$ representing healthy cells. The release of DOX from multiple emulsions is based on a $\mathrm{pH}$-dependent mechanism, shown in Fig. 3a.

This idea was realised by introducing pH-responsive biopolymer (sodium carboxymethylcellulose - CMC-Na) into the external phase of multiple emulsions, which bring forth spatial conformational changes influencing drug release. ${ }^{31}$ As the biopolymer (CMC-Na) is an adhesive polymer, its molecules are present not only in the external phase but also on the surfaces of drops. ${ }^{32}$ As shown in Fig. 3a, under lower pH conditions, the chains of CMC-Na are coiled and form aggregates that interact with one another weakly, facilitating drug release. At a higher or neutral $\mathrm{pH}$, the polymer stretches to form long-loose chains, which interact strongly, leading to overlapping and ultimately hindering the drug release (Fig. 3 a). The spatial conformation changes in polymer chains also promote changes in some of its physicochemical properties. ${ }^{31}$ Our measurements demonstrated differences between viscosities under acidic and slightly base $\mathrm{pH}$ levels for emulsions and their external phases (Fig. $3 \mathrm{~b}, \mathrm{c}$ ). Moreover, viscosity changes affect the values of the diffusion coefficient of the drug. Due to the low concentration of Na-CMC (0.2 wt. \%) the changes in the viscosity were small but significant enough to observe the difference in the diffusional transport of DOX both within and out of the drops (Table 3). The drug release rates also depend on the amount of its elimination by cancer cells. Drug elimination is determined by its physicochemical properties, formulation, type of the cells, route of administration, and rate of drug transport to the brain. In general, drugs can be eliminated including mechanisms such as degradation, metabolism, permeation, local internalisation or binding, and through blood capillaries. ${ }^{33}$ The drug (DOX) depletion due to elimination by GBM cells was determined based on the experimental data of the mass fraction of DOX released from emulsions with and without cancer cells (Fig. 2 c, d, e and f, g, h). A more general model of drug binding in the biological tissue was used to determine the elimination rate constant of DOX, according to the kinetics of an irreversible first-order reaction. ${ }^{9}$ On this basis, the elimination rate constants of DOX (k) by LN229 and U87 MG cells for the emulsions DOX-E1 and DOX-E2 were calculated and compared with those for the DOX in the solution representing classical therapy (DOX in a solution), (Tab. 2). The obtained values of (k) have demonstrated that exposing cancer cells to DOX in emulsions led to higher drug elimination, as compared to classical therapy. Higher values of the consumption rate constants of DOX in emulsion implied a greater effectiveness of the emulsion form of the drug administration, which was confirmed by cytotoxicity studies of both forms of the drug (DOX in emulsion and DOX in a solution). The experimentally obtained values of the drug elimination rate constants (k) were further used in simulations. The simulations of the drug (DOX) release in the GBM cells environment were based on a diffusion model with a chemical reaction (representing elimination/consumption of the DOX by cancer cells). Model equations (eqs. 1-13) were solved for parameters $D_{32}, d_{32}, k_{L} a, \varphi, D_{e}$, $\mathrm{D}_{\mathrm{e}, \mathrm{z}}, \mathrm{k}$, calculated based on the experimental data (Table 3) to find the spatiotemporal drug concentration distribution and then averaged to a defined volume for simulating the cumulative mass of DOX released vs time in the presence of GBM cells. The results of the release rates simulations in comparison to experiments for both investigated emulsions and cell lines (LN229 and U87 MG cells) are presented in Fig. 2 (c, d, f, g). Simulations predicting release rates of DOX from the emulsion implant showed good agreement with experimental data. These results proved that the developed model may be a vital tool in the planning and evaluating brain oncotherapy. One of the stages is, among others, determining the dose and duration of therapy and whether and when the implant should be replaced with a new portion of the emulsion - a new implant. Then, based on the simulation results, it is possible to determine the time of the complete release of the drug and thus the time after which it is necessary to replace the inserted implant with a new portion of the emulsion, i.e. the time when the consumption of DOX by the cells ceases to increase or increases insufficiently. Moreover, by having a process model, the prediction of the drug release rates in the tumour cellular environment is feasible over a longer time scale, concerning the treatment time, compared to experimental studies. In vitro studies of the DOX release from the emulsion in the presence of cells were carried out for a maximum of $24 \mathrm{~h}$ due to the specific nature and limitations of tests with biological material 
related to the cell-culturing under conditions of anti-cancer therapy. The use of this model avoids long-term and costly experimental studies that require work with highly toxic chemotherapeutic agents and biological material. In addition, having then basic data on cell viability (from cytotoxicity study) connected with drug elimination, the effectiveness of the therapy may be easily evaluated based on the model predictions.

3.2 The modelling of the spatiotemporal drug concentration distribution within drops of the emulsion implant and external environment with glioblastoma cells

The numerical simulations also included the spatial concentration distribution of DOX within drops and the surrounding fluid with cancer cells LN229 at a given time for emulsion DOX-E1 (Fig. 4a) and DOX-E2 (Fig. 4b). As shown in exemplary simulations in Fig 4, the concentration of DOX released outside the drop, in the presence of GBM cells, achieved a relatively fast (within 50 seconds) and practically constant value within an already small distance from the surface of the implant (representative drop of emulsion) and within the bulk phase. This is the advantage of the drug emulsion-based implant, which ensures a constant concentration of the drug in the tumour environment controlled by emulsion structures and drop sizes. Simulations enable the prediction of the required drug concentration, and thus the dose of the anti-cancer drug for any cancer cells based on comparing the concentration distribution of DOX within the emulsion implant and the concentration of DOX in the cancer cells environment (external fluid). As shown in Fig. 4 (a, b), despite the same dose of DOX in both emulsions, the gradient of DOX in emulsions DOX-E2 (containing smaller drops) changes faster, due to faster diffusional release, providing smaller values of the drug concentration within and outside the drop, compared to emulsion DOX-E1 (with larger drops) at the same release time. This feature of the emulsion-based implant as a local drug delivery system may be important for planning individualised and tailored drug dosing, and also for achieving the desired therapeutic efficacy and avoiding undesired effects.

\subsection{Cytotoxicity of the drug encapsulated in multiple emulsions for GBM cell lines}

The in vitro cytotoxicity of the multiple emulsions with and without encapsulated DOX, and DOX in a solution (classical chemotherapy), were tested in the presence of glioblastoma multiforme (GBM) cell lines: U87 MG and LN229, after different contact times (24h, 48h, 72h) with cells (Fig. 5).

Firstly, the cytotoxicity of the emulsion without DOX (emulsions E1 and E2) was verified. Reduction in the viability of cells after $72 \mathrm{~h}$ contact with the emulsion was observed (for $87 \mathrm{MG}$ max. to $55 \%$, Fig. 5 a-d; for LN229 max to $45 \%$ Fig. 5 e-h), but this effect disappeared within a maximum of 7 days cell culturing after removal of the emulsion. Secondly, the cytotoxic effect of DOX introduced as an emulsion implant was compared with DOX in a solution (classical therapy) for GBM cells of both lines, and two doses of DOX (0.1 $\mu \mathrm{M}$ and $0.2 \mu \mathrm{M})$. At the lower dose of DOX $(0.1 \mu \mathrm{M})$, a reduction in cell viability within $24-72 \mathrm{~h}$ for both routes of the drug administration was observed in the range: (i) U87 MG to 15-69\% for DOX in emulsions, and to $58-77 \%$ for DOX in solution (Fig. 5 a, c), (ii) LN229 to 39-69\% for DOX in emulsion, and to 54-67\% for DOX in solution (Fig. 5 e, g). Whereas at the higher dose of DOX $(0.2 \mu \mathrm{M})$, a reduction in cell viability was in the range: (i) U87 MG to $25-71 \%$ for DOX in emulsions and to $56-70 \%$ for DOX in solution (Fig. 5 b, d), (ii) LN229 to $36-60 \%$ for DOX in emulsion and to $27-54 \%$ for DOX in solution (Fig. $5 \mathrm{f}, \mathrm{h}$ ). For the tested doses of DOX, a greater cytotoxic effect of DOX in emulsions (max. reduction by 85\%) was observed compared to DOX in a solution (max. reduction by $43 \%$ ) for $0.1 \mu \mathrm{M}$ DOX in emulsion DOX-E1, and U87 MG cell line. These results revealed that DOX delivered as an emulsion provides a greater cytotoxic effect, and therefore increases the efficacy of the therapy compared to classical chemotherapy. Also, these results confirmed the significance of the routes of drug administration for the final effect of this therapy. Our previous studies with a wider range of DOX concentration $(0.01-1 \mu \mathrm{M})$ for U87 MG cells also demonstrated greater effectiveness of DOX therapy based on a drug in an emulsion compared with the classical drug administration. ${ }^{26}$ In addition, DOX in emulsion-based therapy was shown to be effective for the lowest doses of DOX in the range studied, which were ineffective with the classical therapy (DOX solution). Next, the cytotoxic effect between the emulsion form of DOX (DOX-E1 and DOX-E2) was compared. In case of line U87 MG cells, cell viability after DOX administration in emulsion were (i) for DOX-E1: $15-57 \%$ for the 0.1 $\mu \mathrm{M}$ DOX dose and 25-63\% for the $0.2 \mu \mathrm{M}$ DOX dose (Fig. 5 a-b), and (ii) for DOX-E2: $50-69 \%$ for the 0.1 $\mu \mathrm{M}$ DOX dose and $46-71 \%$ for the $0.2 \mu \mathrm{M}$ DOX dose (Fig. 5 c-d). For the LN229 cell line, cell viability after 
DOX administration in emulsion achieved (i) for DOX-E1: $36-46 \%$ for the $0.1 \mu \mathrm{M}$ DOX dose and $36-49 \%$ for the $0.2 \mu \mathrm{M}$ DOX dose (Fig. 5 e-f), and (ii) for DOX-E2: $53-69 \%$ for the $0.1 \mu \mathrm{M}$ DOX dose and $48-60 \%$ for the $0.2 \mu \mathrm{M}$ DOX dose (Fig. $5 \mathrm{~g}$-h). Emulsion DOX-E1 demonstrated greater efficacy of reduction in viability of the tested GBM cell lines compared to DOX-E2. The DOX-E1 and DOX-E2 systems differed in the drop sizes and internal structures, as well as in the composition of the external phases, and the volume fraction of the dispersed phases. Differences between emulsions DOX-E1 and DOX-E2 affected the release rates of DOX, which contributed to the different efficacy in reducing cancer cell viability. More cytotoxic and thus effective was emulsion DOX-E1 with larger membrane phase drops with a structure of many small internal droplets, from which DOX release is slower than from emulsion DOX-E2. This proved that the drug release rate from a delivery system has a direct impact on cancer cell viability and thus on the final therapeutic effect.

\section{CONCLUSIONS}

This paper presents results of a promising strategy for the efficient local delivery of an anti-cancer drug (doxorubicin-DOX) to brain tumours based on an injectable three-phase liquid implant in the form of $\mathrm{W} 1 / \mathrm{O} / \mathrm{W} 2$ multiple emulsion. The multiple emulsions have structures of droplets in drops, therefore protecting healthy cells by encapsulating an aggressive anti-cancer drug within their internal droplets surrounded by larger drops of patient-friendly oil. The drug is gradually released by diffusion at predetermined rates from the internal droplets of the emulsions-based implant containing biopolymer (sodium carboxymethylcellulose) in response to the acidic tumour microenvironment, and is then transported to the tumour. The implant was designed to sustainably deliver therapeutics for up to 100 hours or longer, depending on the parameters of the emulsion: drop sizes, structure, viscosity, and the encapsulated drug dose. The comprehensive experimental study included drug release, in the presence and absence of glioblastoma multiforme (GBM) cell lines (LN229, U87 MG), and cell viability to evaluate the effectiveness of the proposed therapy. Also, a diffusion-reaction model has been adapted to analyse and predict doxorubicin release kinetics and drug elimination by glioblastoma cells to evaluate the proposed therapy. The model equations include parameters that take into account the structure of the emulsion (drop size and packing volume fraction), drug absorption (elimination rate constant) by cancer cells, and drug diffusion coefficients inside the emulsion drops and in the tumour environment. Drug elimination was modelled assuming first-order reaction kinetics. The numerical simulations of the drug concentration distribution in time and space were performed for the release process from emulsions DOX- E1 and DOX-E2 in the presence of glioblastoma cells (U87 MG and LN229) according to model parameters based on the experimental data. The CFD numerical simulation confirmed that the drug release process is controlled by the parameters of the emulsion structures. The obtained fractional release of the chemotherapeutic drug showed a faster release rate, from the emulsion DOX-E2, of smaller drops (higher interfacial area) compared to the emulsion DOX-E1 (bigger drops - smaller interfacial area) in the presence of the tested cancer cell lines. In addition, simulations of the spatiotemporal drug concentration distribution outside the drops of the emulsion implant confirmed a constant drug concentration, close to the implant surface and in the bulk phase, essential for the effectiveness of the therapy. Maintaining a constant concentration of the drug in the cancer cells environment confirmed the advantage of multiple emulsion as an implant delivering the drug. The validation of this model by comparison with experimental data showed good agreement under a variety of conditions (emulsions drop sizes and structures: DOX-E1 and DOX-E2, DOX concentration and types of cancer cells). The best effectiveness of the therapy was experimentally confirmed by a significant reduction in the GBM cell viability by $85 \%$ for emulsion DOX-E1, whereas for DOX in a solution (classical chemotherapy) by $43 \%$ depending on the dose of the drug. This was also confirmed by the higher drug elimination rate constants by cancer cells treated with a chemotherapeutic in a multiple emulsion compared to classical chemotherapy. Moreover, emulsion-based therapy, even with the lowest dose of DOX responded with reduced cell viability, whereas for the traditionally delivered drug this dose was ineffective. The obtained results showed some considerable promise and proved that emulsionbased implant anti-cancer drugs delivery might succeed in the unequal fight against brain tumours. The adapted diffusion-reaction model has several potential uses, especially in early pre-clinical development in the planning of optimal oncotherapy, including drug dose and release kinetics and treatment duration. This 
model, supported by experimental data, also provides insights into the relevant mechanisms and parameters, which quantitatively describe the complex processes accomplished by drug release in a biological system.

\section{ACKNOWLEDGMENT}

Research was funded by (POB Biotechnology and Biomedical Engineering) of Warsaw University of Technology within the Excellence Initiative: Research University (IDUB) programme (project BIOTECHMED-2) and by the National Science Centre - Poland (grant number: 2014/13/B/ST8/04274).

\section{REFERENCES}

1. Lu CT, Zhao YZ, Wong HL, Cai J, Peng L, Tian XQ. Current approaches to enhance CNS delivery of drugs across the brain barriers. Int J Nanomedicine. 2014;9:2241-2257.

2. Tan AC, Ashley DM, López GY, Malinzak M, Friedman HS, Khasraw M. Management of Glioblastoma: State of the Art and Future Directions. CA Cancer J Clin. 2020;70:299-312.

3. Kasinathan N, Jagani HV, Alex AT, Volety SM, Rao JV. Strategies for drug delivery to the central nervous system by systemic route. Drug Deliv. 2015;22:243-257.

4. Chakroun RW, Zhang P, Lin R, Schiapparelli P, Quinones-Hinojosa A, Cui H. Nanotherapeutic systems for local treatment of brain tumors. WIREs Nanomed Nanobiotechnol. 2018;10(1):e1479.

5. Haumann R, Videira JC, Kaspers GJL, van Vuurden DG, Hulleman E. Overview of Current Drug Delivery Methods Across the Blood-Brain Barrier for the Treatment of Primary Brain Tumors. CNS Drugs. 2020;34:1121-1131.

6. Mueller E, Himbert S, Simpson MJ, Bleuel M, Rheinstadter MC, Hoare T. Cationic, Anionic, and Amphoteric Dual pH/Temperature-Responsive Degradable Microgels via Self-Assembly of Functionalized Oligomeric Precursor Polymers. Macromolecules. 2021;54(1):351-363.

7. Gerlee P. The model muddle: in search of tumor growth laws. Cancer Res. 2013;73(8):2407-2411.

8. Preziosi L. Cancer modelling and simulation (1st edition). Chapman \& Hall, CRC, 2003.

9. Weiser JR, Saltzman WM. Controlled release for local delivery of drugs: barriers and models. J Control Release. 2014;190:664-673.

10. Siepmann J, Siepmann F. Modeling of diffusion controlled drug delivery. J Control Release. 2012;161(2):351-362.

11. Kim M, Gillies RJ, Rejniak KA. Current advances in mathematical modeling of anti-cancer drug penetration into tumor tissues. FRONT ONCOL. 2013;3:278.

12. Montigny JD, Iosif A, Breitwieser L, Manca M, Bauer R, Vavourakis V. An in silico hybrid continuum/agent-based procedure to modelling cancer development: interrogating the interplay amongst glioma invasion, vascularity and necrosis. Methods. 2020;185(4):94-104.

13. Chen J, Weihs D, Vermolen FJ. A Cellular Automata Model of Oncolytic Virotherapy in Pancreatic Cancer. Bull Math Biol. 2020;82(103):1-25.

14. Metzcar J, Wang Y, Heiland R, Macklin P. A Review of Cell-Based Computational Modeling in Cancer Biology. JCO Clin. Cancer Inform. 2019;3(3):1-13.

15. Trucu D, Lin P, Chaplain MA, Wang Y. A multiscale moving boundary model arising in cancer invasion. Multiscale Model. Simul. 2013;11(1):309-335.

16. Ramis-Conde I, Chaplain MAJ, Anderson ARA. Mathematical modelling of cancer cell invasion of tissue. Math. Comput. Modelling. 2008;47:533-545.

17. Dluska E, Hubacz R, Wronski S, Kamienski J, Dylag M, Wojtowicz R. The influence of helical flow on water fuel emulsion preparation. Chem. Eng. Commun. 2007;194(10): 1271-1286.

18. Dluska E, Markowska-Radomska A, Metera A, Kosicki K. Hierarchically structured emulsions for brain therapy. Colloid Surf. A Physicochem. Eng. Asp. 2019;575:205-211.

19. Eugenia Carlotti M, Gallarate M, Sapino S, Ugazio E, Morel S. W/O/W Multiple Emulsions for Dermatological and Cosmetic Use, Obtained with Ethylene Oxide Free Emulsifiers. J Dispers Sci Technol. 2005;26(2):183-192.

20. Loya-Castro MF, Sanchez-Mejia M, Sanchez-Ramirez DR, Dominguez-Rios R, Escareno N, OcegueraBasurto PE, Figueroa-Ochoa EB, Quintero A, del Toro-Arreola A, Topete A, Daneri-Navarro A. Prepa- 
ration of PLGA/Rose Bengal colloidal particles by double emulsion and layer-by-layer for breast cancer treatment. J. Colloid Interface Sci. 2018;518:122-129.

21. Dluska E, Metera A, Markowska-Radomska A, Tudek B. Effective cryopreservation and recovery of living cells encapsulated in multiple emulsions. Biopreserv Biobank. 2019;17(5):468-476.

22. Mutaliyeva B, Grigoriev D, Madybekova G, Sharipova A, Aidarova S, Saparbekova A, Millere R. Microencapsulation of insulin and its release using w/o/w double emulsion method. Colloids Surf. A Physicochem. Eng. Asp. 2017;521:147-152.

23. Bozkir A, Hayta G. Preparation and evaluation of multiple emulsions water-in-oil-in-water (w/o/w) as delivery system for influenza virus antigens. J. Drug. Target. 2004;12:157-164.

24. Soriano-Ruiz JL, Suner-Carbo J, Calpena-Capmany AC, Bozal-de Febrer N, Halbaut-Bellowa L, BoixMontanes A, Souto EB, Clares-Naveros B. Clotrimazole multiple W/O/W emulsion as anticandidal agent: Characterization and evaluation on skin and mucosae. Colloids Surf. B Biointerfaces. 2018;175:166-174.

25. McClements DJ, Decker EA, Weiss J. Emulsion-based delivery systems for lipophilic bioactive components. J Food Sci. 2007;72(8):109-124.

26. Dluska E, Markowska-Radomska A, Metera A, Tudek B, Kosicki K. Multiple emulsions as effective platforms for controlled anti-cancer drug delivery. Nanomedicine. 2017;12(18):2183-2197.

27. Dluska E, Cui Z, Markowska-Radomska A, Metera A, Kosicki K. Cryoprotection and banking of living cells in a 3D multiple emulsion-based carrier, Biotechnology Journal. 2017;12(8):1-7.

28. Dluska E, Markowska-Radomska A. Regimes of multiple emulsions of W1/O/W2 and O1/W/O2 type in the continuous Couette-Taylor flow contactor. Chem. Eng. Technol. 2010;33(1):113-120.

29. Markowska-Radomska A, Dluska E. An evaluation of a mass transfer rate at the boundary of different release mechanisms in complex liquid dispersion. Chem Eng Process. Process Intensification. 2016;101:56-71.

30. Markowska-Radomska A, Dluska E. The Multiple Emulsion Entrapping Active Agent Produced via One-Step Preparation Method in the Liquid-Liquid Helical Flow for Drug Release Study and Modeling. Progr Colloid Polymer Sci. 2012;139:29-34.

31. Khaled B, Abdelbaki B. Rheological and electrokinetic properties of carboxymethylcellulose-water dispersions in the presence of salts. Int. J. Phys. Sci. 2012;7(11):1790-1798.

32. Friedman D, Schwartz J, Amselem S. Bioadhesive emulsion preparations for enhance drug delivery. US Patent Number: USOO5744155A, 1998.

33. Weiser JR, Saltzman WM. Controlled release for local delivery of drugs: barriers and models. J Control Release. 2014;190:664-673.

34. Li J, Carr PW. Accuracy of empirical correlations for estimating diffusion coefficients in aqueous organic mixtures. Anal. Chem. 1997;69:2530-2536.

35. Bartzatt R. Identification of doxorubicin and an imine derivative from liquid and solid samples utilizing liquid chromatography. J Liq Chromatogr Relat Technol. 2006;29:2303-2312.

36. Yan N. A mass transfer model for type-II-facilitated transport in liquid membranes. Chem. Eng. Sci. 1993;48:3835-3843.

37. Lian G, Malone ME, Homan JE, Norton IT. A mathematical model of volatile release in the mouth from the dispersion of gelled emulsion particles. J. Control. Release. 2004;98:139-155.

38. Happel J. Viscosity of suspensions of uniform spheres. J. Appl. Phys. 1957;28:1288-1292.

\section{Hosted file}

Table 1.docx available at https://authorea.com/users/422092/articles/527851-mass-transferof-anti-cancer-drug-delivery-to-brain-tumours-by-a-multiple-emulsion-based-implant

\section{Hosted file}

Table 2.docx available at https://authorea.com/users/422092/articles/527851-mass-transferof-anti-cancer-drug-delivery-to-brain-tumours-by-a-multiple-emulsion-based-implant

\section{Hosted file}


Table 3.docx available at https://authorea.com/users/422092/articles/527851-mass-transferof-anti-cancer-drug-delivery-to-brain-tumours-by-a-multiple-emulsion-based-implant

I. Detection of the tumor

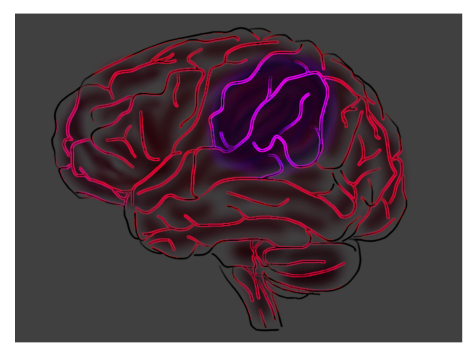

II. Surgical removal of the tumor

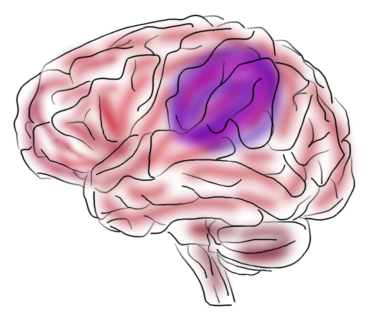

III. Intraoperative insertion of the emulsion implant into the tumor resection cavity

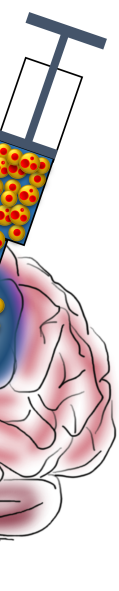

EMULSION IMPLANT for delivery of drug (doxorubicin)

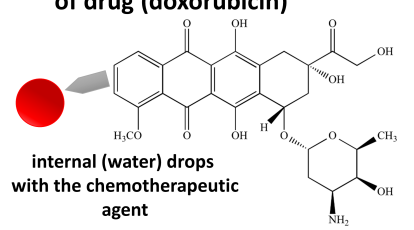


(a)

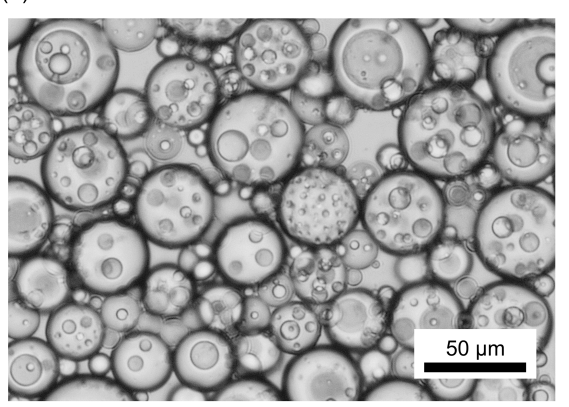

(c)

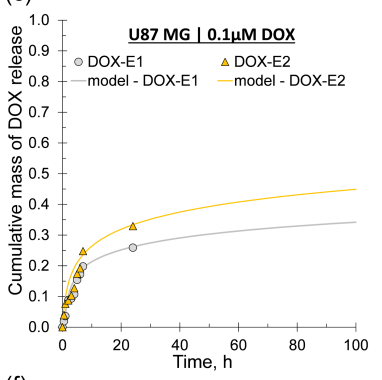

(f)

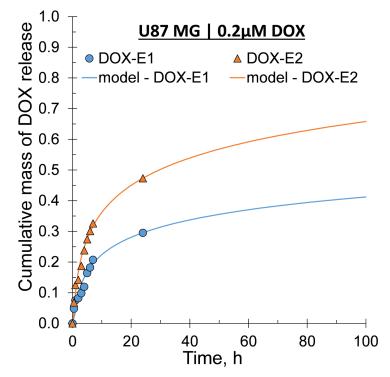

(b)

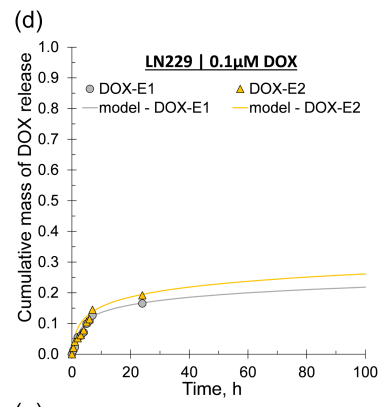

(g)

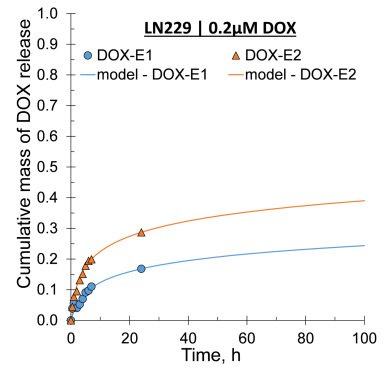

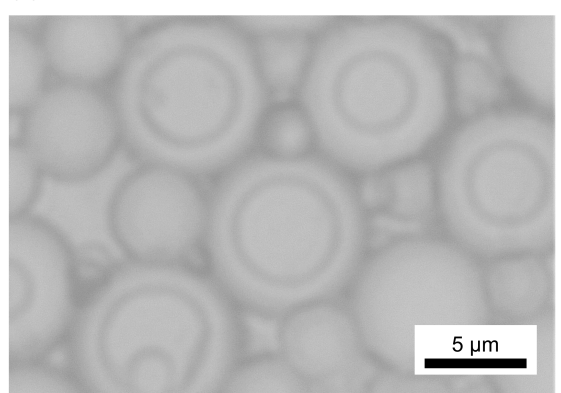

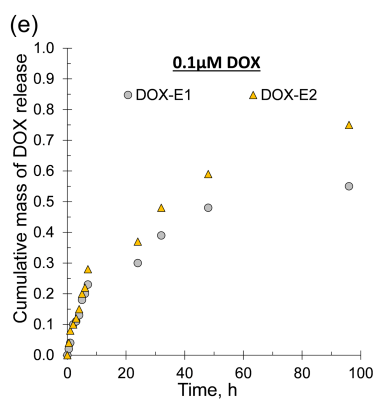

(h)

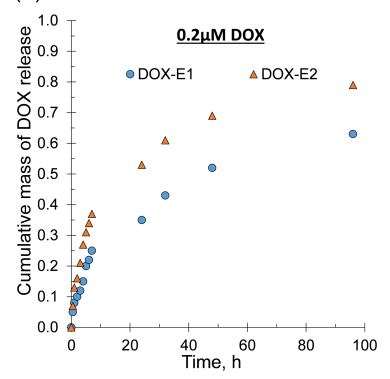


(a)
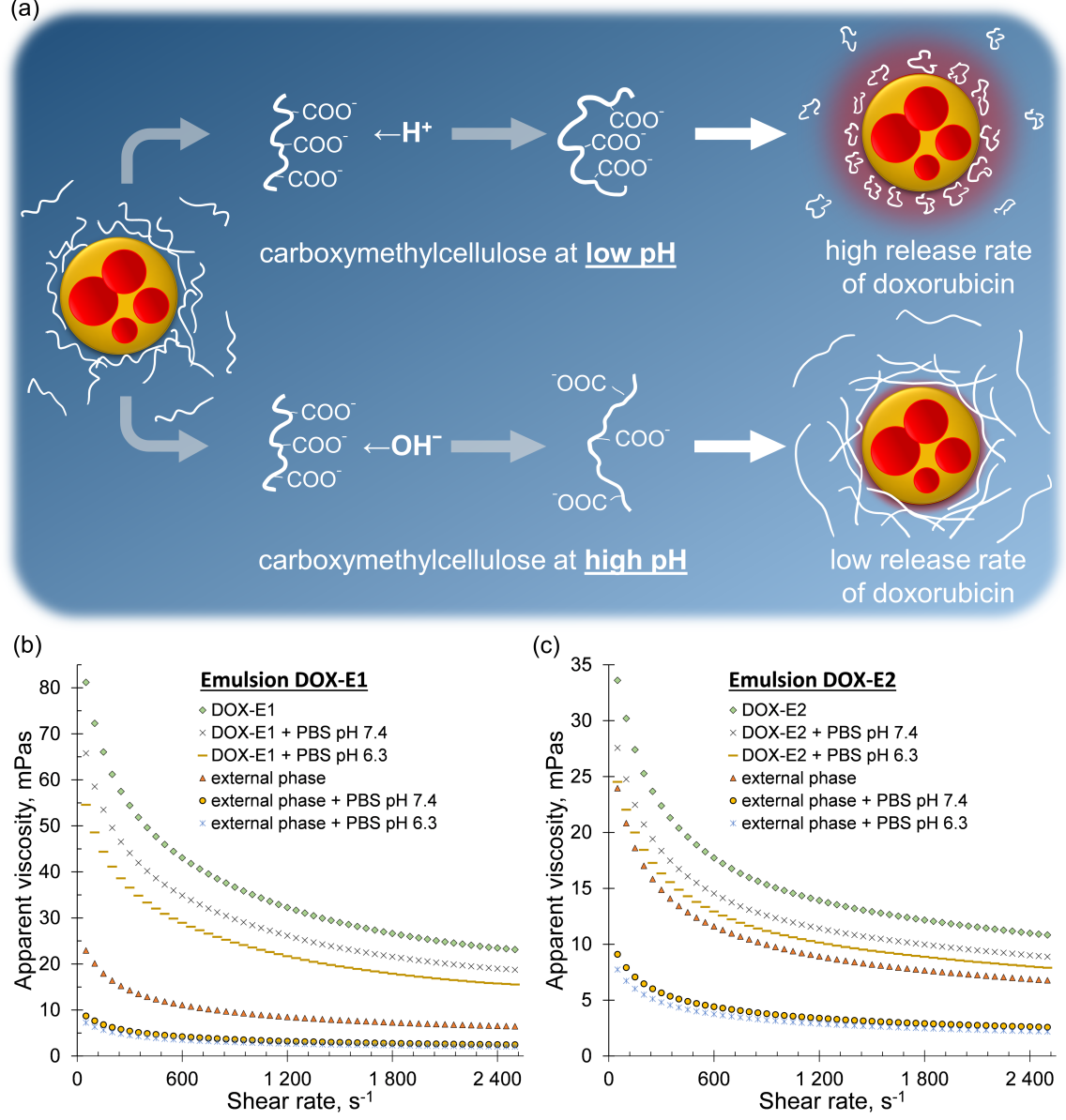
(a)

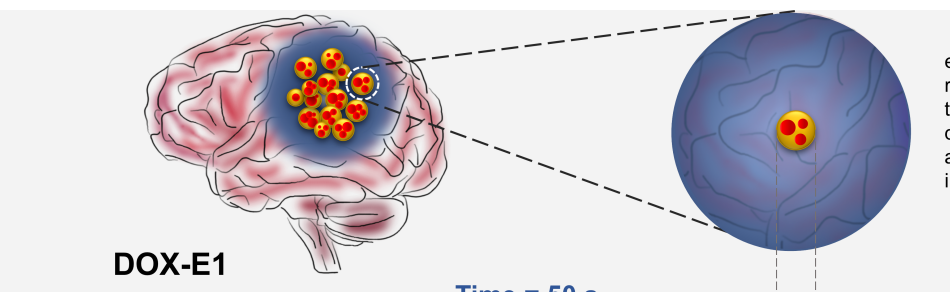

emulsion drop

representative for

the population of

drops constituting

an emulsion

implant

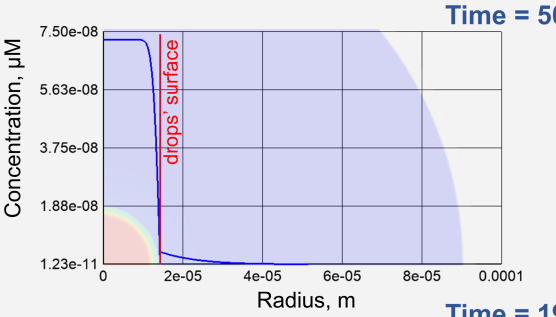

ime $=\mathbf{5 0 ~ s}$
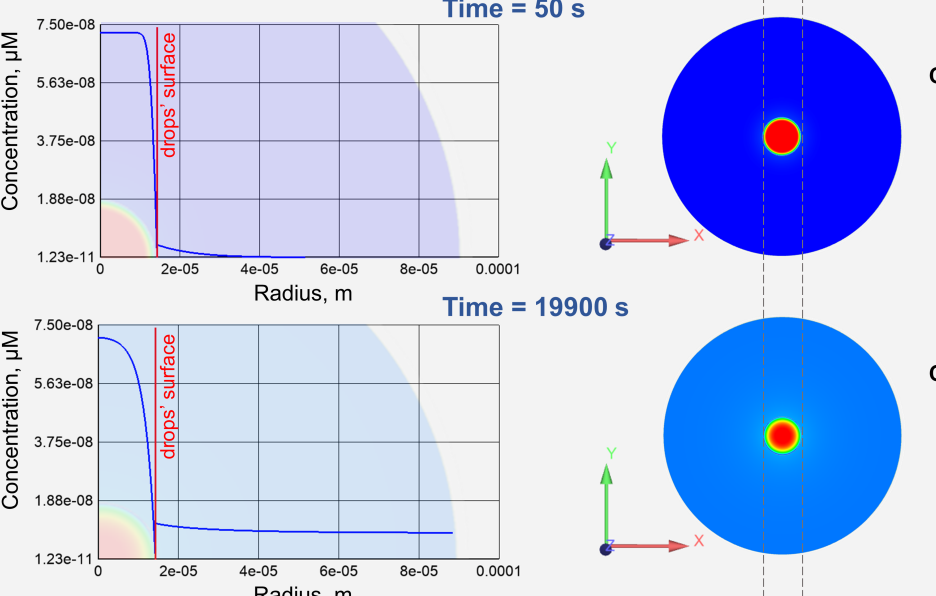

Concentration

$(\mu \mathrm{M})$

6.95e-08

$5.21 \mathrm{e}-08$

$3.47 \mathrm{e}-08$

$1.74 \mathrm{e}-08$

$1.23 \mathrm{e}-11$

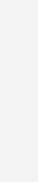

Time $=41500 \mathrm{~s}$
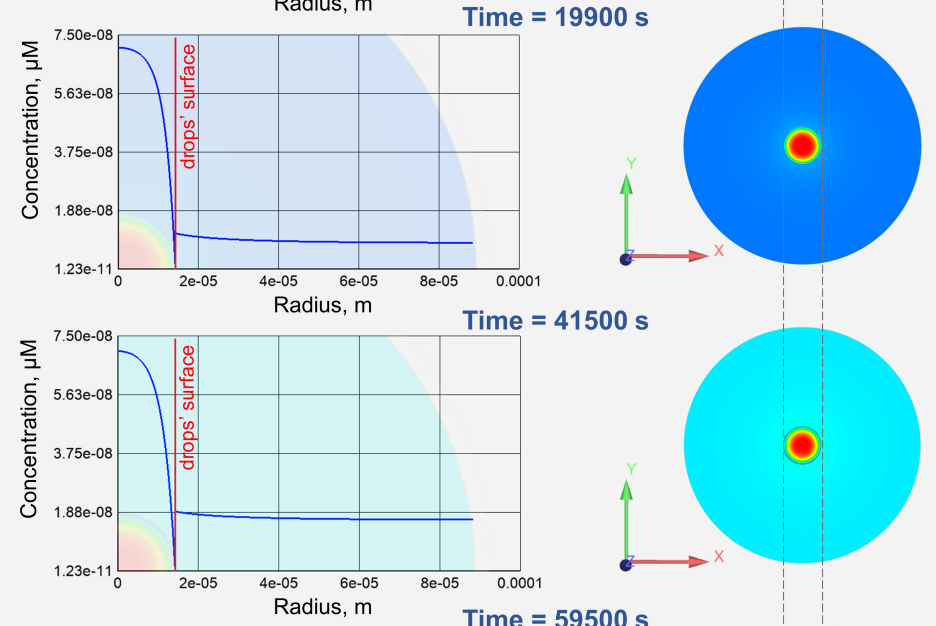

Concentration

$(\mu \mathrm{M})$

$6.95 \mathrm{e}-08$

5.21e-08

$3.47 \mathrm{e}-08$

$1.74 \mathrm{e}-08$

$1.23 \mathrm{e}-11$
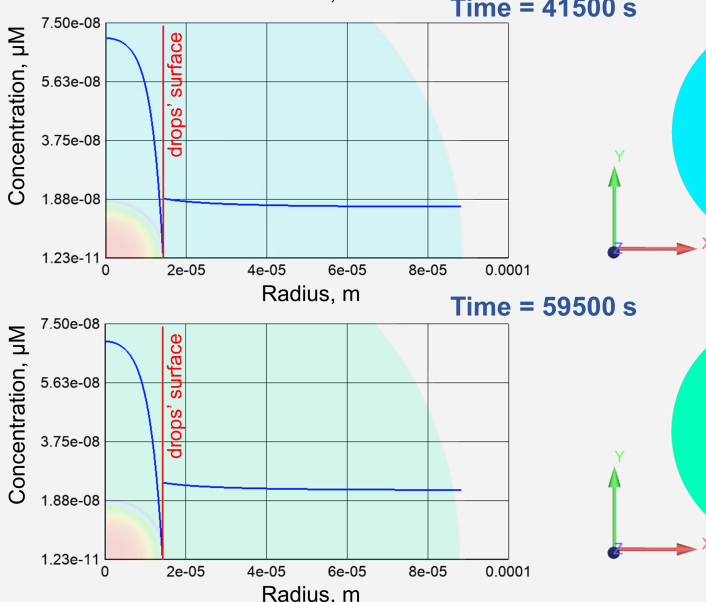

Concentration

$(\mu \mathrm{M})$

$6.95 \mathrm{e}-08$

$5.21 \mathrm{e}-08$

$3.47 \mathrm{e}-08$

$1.74 \mathrm{e}-08$

$1.23 \mathrm{e}-11$

Concentration

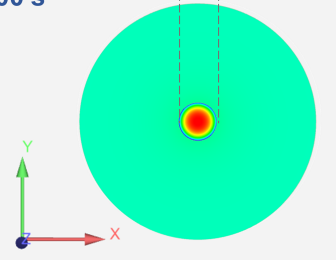

$(\mu \mathrm{M})$

$6.95 \mathrm{e}-08$
$5.21 \mathrm{e}-08$

$3.47 \mathrm{e}-08$

$1.74 \mathrm{e}-08$

$1.23 \mathrm{e}-11$ 
(b)

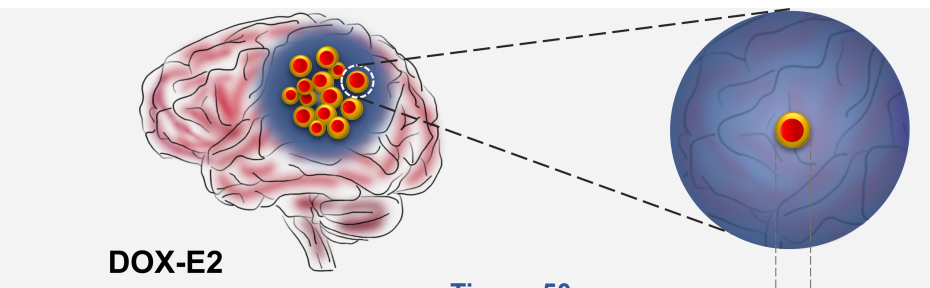

emulsion drop

epresentative for

the population of

drops constituting

an emulsion

implant
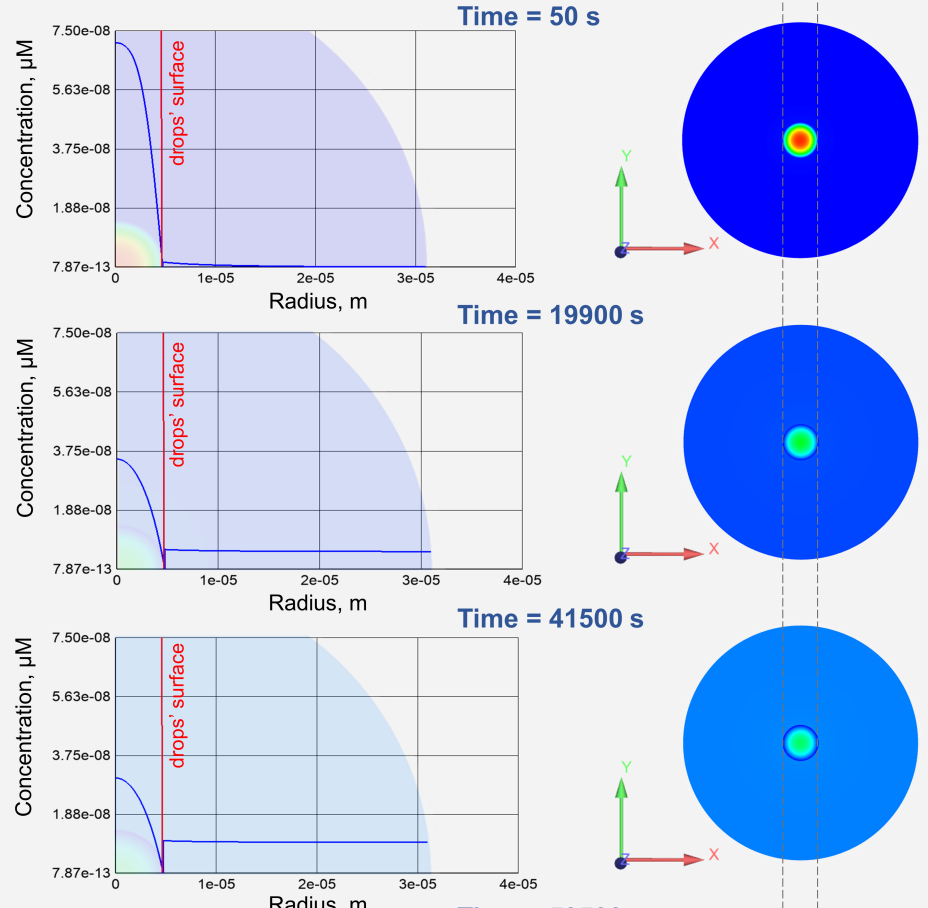

Concentration $(\mu \mathrm{M})$

$7.50 \mathrm{e}-08$
$5.63 \mathrm{e}-08$

$3.75 \mathrm{e}-08$

$1.88 \mathrm{e}-08$

$7.87 \mathrm{e}-13$
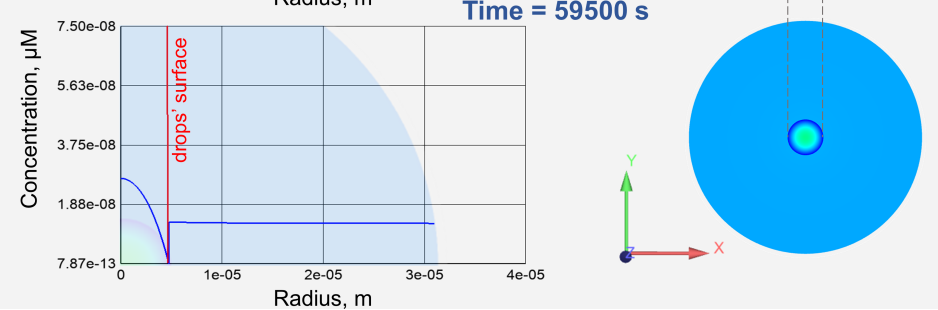

Concentration

$(\mu \mathrm{M})$

$7.50 \mathrm{e}-08$

$5.63 \mathrm{e}-08$

$3.75 \mathrm{e}-08$

$1.88 \mathrm{e}-08$
$7.87 \mathrm{e}-13$ 

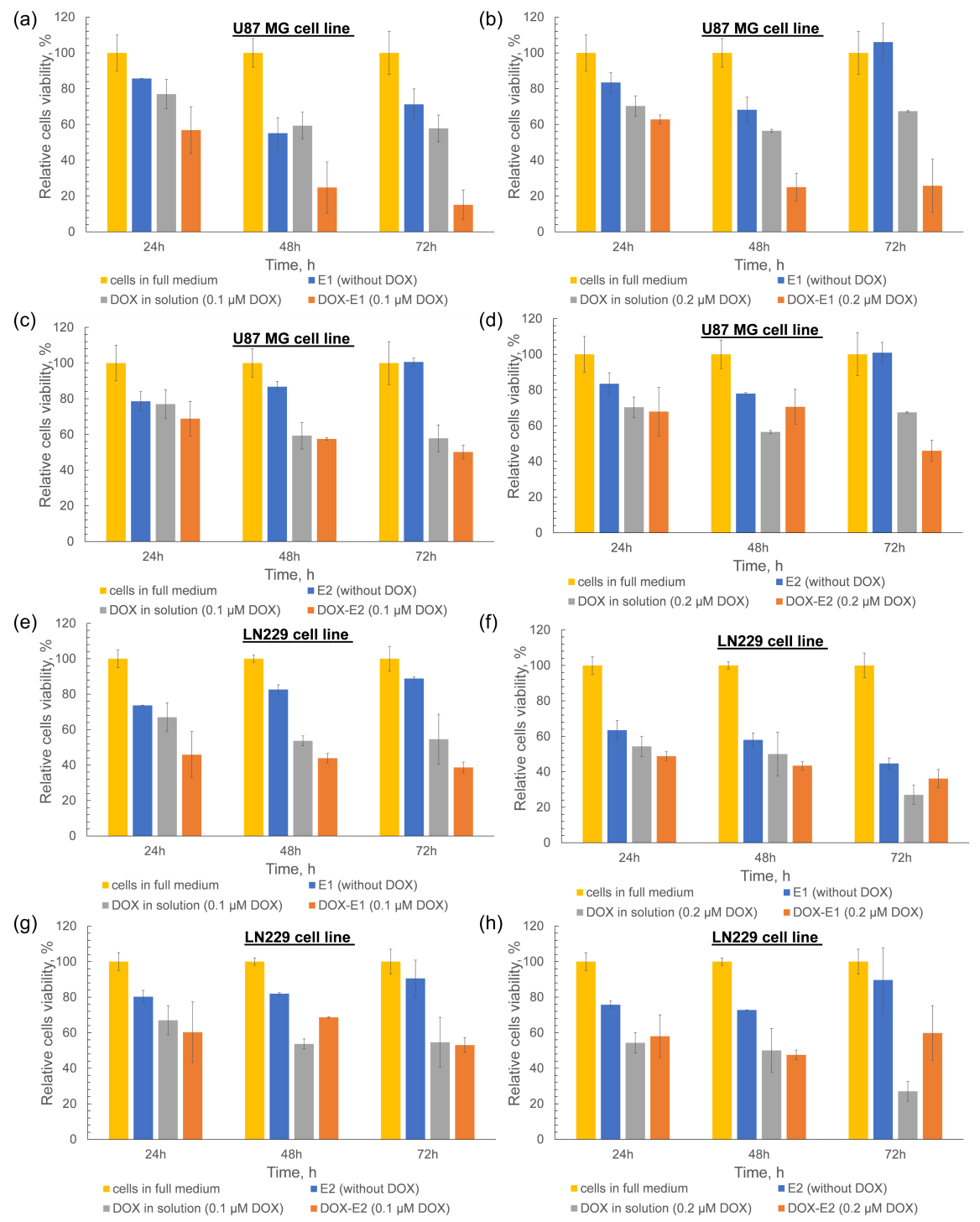